\title{
The influence of numerical advection schemes on the results of ocean general circulation models*
}

\author{
Rüdiger Gerdes**, Cornelia Köberle, and Jürgen Willebrand \\ Institut für Meereskunde an der Universität Kiel, Düsternbrooker Weg 20, W-2300 Kiel 1, FRG \\ Received January 3, 1990/Accepted November 16, 1990
}

\begin{abstract}
The dependence of results from coarse-resolution models of the North Atlantic circulation on the numerical advection algorithm is studied. In particular, the sensitivity of parameters relevant for climate simulations as e.g., meridional transport of mass and heat and main thermocline thickness is investigated. Three algorithms were considered: (a) a central difference scheme with constant values for horizontal and vertical diffusion, (b) an upstream scheme with no explicit diffusion, and (c) a flux-corrected transport (FCT) scheme with constant and strictly isopycnal diffusion. The temporal evolution of the three models on time scales of centuries is markedly different, the upstream scheme resulting in much shorter adjustment time whereas the central difference scheme is slower and controlled by vertical diffusion rather than advection. In the steady state, the main thermocline structure is much less diffusive in the FCT calculation which also has much lower heat transport. Both horizontal circulation and overturning in the meridional-vertical plane are strongest in the upstream-model. The results are discussed in terms of the effective vertical (diapycnal) mixing in the different models. A significant increase in vertical resolution would be required to eliminate the high sensitivity due to the numerical algorithms, and allow physically motivated mixing formulations to become effective.
\end{abstract}

\section{Introduction}

The coupling of ocean and atmosphere through heat and fresh water fluxes is an essential factor for climate

* This paper was presented at the International Conference on Modelling of Global Climate Change and Variability, held in Hamburg 11-15 September 1989 under the auspices of the Meteorological Institute of the University of Hamburg and the Max Planck Institute for Meteorology. Guest Editor for these papers is Dr. L. Dümenil

** Present address: Geophysical Fluid Dynamics Laboratory, Princeton University, P. O. Box 308, Princeton, NJ 08540, USA

Offprint requests to: $\mathrm{J}$ Willebrand change on time scales of decades and longer. A quantitative understanding of climate dynamics on these time scales requires ocean general circulation models which describe the formation and large-scale transport of water-mass properties with sufficient accuracy.

To account properly for the important influence of eddies on the mean circulation, models with a horizontal resolution smaller than the internal Rossby radius [i.e., $0(30) \mathrm{km}$ ] are required. Although recently progress has been made in this direction (Cox 1985; Holland 1988, personal communication; Semtner and Chervin 1988), for long-term integrations necessary in the context of climate studies, such models are computationally not yet feasible. Models with coarse resolution (typically a few hundred kilometers) which include the effect of eddies parametrically therefore continue to be necessary, in particular, for experiments with coupled ocean-atmosphere models. At these scales the momentum balance is very nearly geostrophic, and effects of friction and nonlinearity are normally assumed to be unimportant outside certain boundary layers. Some circulation models have been constructed based on this approximation (Hasselmann 1982; Colin de Verdiere 1988), although traditionally most models have employed the primitive equation system (e.g., Bryan and Lewis 1979).

The evolution of temperature and salinity is affected by the advecting current field, and by mixing processes of various kinds. In the main thermocline advection is dominant, and important dynamic aspects of the horizontal gyre circulation can be understood using simplified advective models (e.g., Luyten et al. 1983; Rhines and Young 1982). However, as demonstrated by F. Bryan (1987) the model response to thermohaline forcing on the very long overturning and diffusive timescales depends crucially on the strength of diapycnal mixing. In particular, this is true for the rate of vertical overturning and deep water formation, the meridional transport of heat, and the thickness of the main thermocline. Isopycnal mixing through stirring by geostrophic eddies predominantly affects temperature and salinity distribution on isopycnals, but can also be 
of dynamic importance through the nonlinearity in the equation of state.

The dependence on the diapycnal mixing is troublesome with reference to our ability to model the oceanic response to climatic change for at least two reasons. One is that the representation of subgridscale processes appropriate for large-scale models is not well understood (see e.g., Holloway 1989, for a recent discussion). So far, empirical parameterizations have been used, employing e.g., constant diffusion coefficients, or prescribed vertical profiles, or formulations depending on stability (Gargett 1984) or Richardson-number (Pacanowski and Philander 1981). The other reason is that even if the effect of subgridscale processes were adequately known, we might not be able to represent them in our models because of numerical limitations. In coarse resolution models there is a close connection between numerical schemes and mixing parametrizations. Some schemes require a certain amount of explicit (but not physically motivated) mixing to maintain numerical stability while others contain mixing in an implicit way. Also, the traditional choice of the diffusion tensor with principal axes parallel to the coordinate directions introduces additional diapycnal diffusion which may not be physically justified.

Part of our motivation in considering the question of numerical advection and mixing algorithms arose from an experiment with the GFDL ocean model described as follows. The vertical mixing rate was set to $0.3 \cdot 10^{-4} \mathrm{~m}^{2} \mathrm{~s}^{-1}$, a typical value for the interior mixing rate as inferred by Olbers et al. (1985). Fig. 1 shows a salinity section at $30^{\circ} \mathrm{W}$ after 620 years of simulated time. The origin of the salinity maximum at the equator was found to be a single gridpoint in the Guinea basin below the sharp halocline associated with river runoff. This sharp front disperses and produces a high salinity anomaly which is physically impossible as it violates the second law of thermodynamics. That signal nevertheless is distributed by the equatorial current system and by diffusion over large distances until it becomes a major water mass in the simulation. The only way to prevent such numerically induced anomalies in a central difference scheme is to increase the mixing, or the resolution. As a much less attractive alternative, one could, of course, also change the boundary conditions which cause the annoying behaviour.

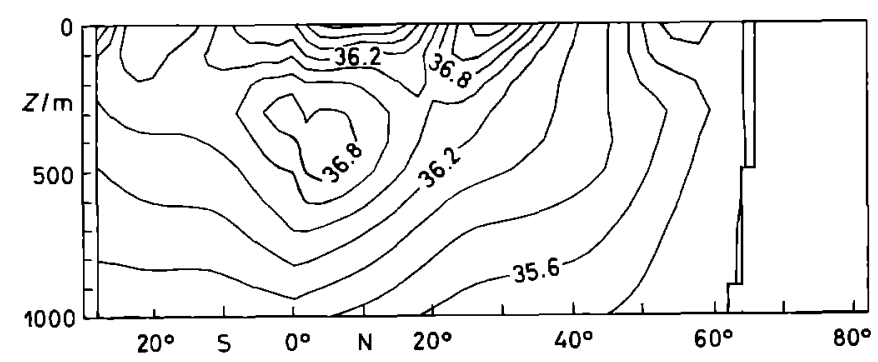

Fig. 1. Salinity section along $30^{\circ} \mathrm{W}$ after 620 years of integration with the GFDL ocean model for an Atlantic configuration. The vertical Austausch is $0.3 \cdot 10^{-4} \mathrm{~m}^{2} / \mathrm{s}$ in this experiment. C.I. 0.2 psu
In our experiments with an Atlantic OGCM we wanted to address the following questions for a special, but perhaps typical, configuration:

1. To what extent are model results sensitive to the choice of the numerical scheme used for the advection of temperature and salinity?

2. How does the mixing, implicit in numerical schemes, vary spatially and how does it compare with the explicit diffusion for the traditional central differences scheme?

3. How are the differences in the results related to the differences in the mixing?

4. Which model compares best with observations and for what reasons?

5. What consequences can be drawn for the implementation of physically motivated mixing schemes in typical large-scale GCMs?

The emphasis of this study is on those aspects of ocean circulation models which are relevant to climate change on decadal and longer time scales, i.e., the formation and distribution of water masses and oceanic heat transport. A discussion of the influence of advection and mixing schemes on gyre-scale motions can be found in Gerdes (1988).

In the second section of this paper, properties of some numerical advection schemes are discussed for an idealized situation, that resembles a particular aspect of the ocean circulation problem. The material in this section is meant to be illustrative rather than to give a thorough mathematical discussion which can be found elsewhere. The third section describes the philosophy and configuration of the numerical experiments with the Atlantic GCM. Results are presented in the fourth section, and a concluding discussion is given in the final section.

\section{Properties of numerical advection algorithms}

The evolution of temperature, salinity and passive tracers in the ocean interior is dominated by advection, although mixing processes are crucial for various aspects of the circulation. An essential requirement for ocean circulation models, therefore, is that the advection process be properly represented. The role of the numerical algorithm on the solution of advection equations has been considered many times (see e.g., Mesinger and Arakawa 1976; O'Brien 1986; Rood 1987 where detailed mathematical analyses and many additional references can be found). The choice between different algorithms involves trade-offs of various kinds. Most of the general discussions regarding the properties of various schemes have been in terms of simplified 1- or 2dimensional systems with little resemblance to ocean circulation problems. In the following, we will give a brief description of a small selection of algorithms which have been used or proposed for ocean circulation models, and illustrate their properties in situations which resemble certain aspects of the propagation of water-mass characteristics in the ocean. For mathemati- 
cal details the reader is referred to the references just cited.

The central difference algorithm is by far the most popular scheme in ocean circulation models. The flux across the boundary of two grid boxes is calculated as the product of normal velocity with an average of both upstream and downstream concentrations. This is combined with a leapfrog (central difference) scheme in time, with an occasional forward timestep to suppress the computational mode. The scheme conserves total mass and is of second-order accuracy (strictly speaking, the latter is true only for uniform grids, and there is some loss of accuracy due to non-uniform vertical resolution in most circulation models). Its main disadvantages is the numerical dispersion that is most noticeable near large gradients in the advected quantity. Non-physical oscillations or "ripples" (under- and overshoots) and negative concentrations of positive definite quantities may occur. Such numerical solutions violate the second law of thermodynamics. An illustration of this numerical error in a circulation model has been already discussed (Fig. 1). Addition of explicit diffusion is required to reduce or eliminate this problem. The required amount of explicit diffusion depends on the actual situation and will be discussed in connection with the following examples.

The upstream scheme has very different numerical errors. Here one-sided upstream differences in each space direction are used to calculate the advective fluxes, in combination with forward time-stepping. The scheme conserves total mass and is of first-order accuracy. It is free from the dispersive effects mentioned above. Its main disadvantages is the large amount of implicit diffusion. For this reason, the upstream scheme has been very unpopular with most ocean modelers. It can be shown, however, that no linear algorithm with less implicit diffusion exists which is strictly free of dispersive effects.

Central differences and upstream algorithms represent, in a sense, opposite extremes, each minimizing one kind of numerical error at the expense of another. A linear compromise between both schemes may be useful in certain cases (e.g., Fiadeiro and Veronis 1977) but will in general exhibit dispersive effects. A nonlinear compromise is the flux-corrected transport (FCT) algorithm (Boris and Book 1973; Zalesak 1979). Here the flux difference (anti-diffusive flux) between a central difference scheme (or any other higher order scheme) and an upstream scheme is computed. Adding the anti-diffusive flux in full to the upstream flux would maximally reduce diffusion but introduce dispersive ripples. The central idea is to limit the anti-diffusive flux locally such that no under- and overshoots are introduced. One possible criterion is e.g., to insist that from one time step to the next no new maxima or minima around one grid cell are created by advection. As remarked by Rood (1987), the FCT is more a philosophy rather than an explicit algorithm, as the crucial limiting step is essentially left to the user's discretion. Depending on the choice of the limiting step the results will be closer to those of either the upstream or the cen- tral differences scheme. The amount of implicit mixing does, therefore, depend on a subjective choice. With this limitation in mind, we may regard the FCT algorithm as a way to find the minimum mixing that is consistent with the thermodynamical constraint. We employ the limiting step described by Zalesak (1979; see Appendix) for all applications of the FCT algorithm discussed in this paper.

As an example to illustrate the properties of those schemes, we consider a situation where a permanent front is maintained by advection of watermasses of different origin, a situation which may have some analogy to the main thermocline in the ocean. We have obtained numerical solutions of the advection equation

$$
\frac{\partial T}{\delta t}-\Psi_{y} T_{x}+\Psi_{x} T_{y}=0
$$

with a stream function given by

$\Psi(x, y)=\sin (\pi y) \cos \{\pi[x+\varepsilon(y-0.5)]\}$

which is shown in Fig. 2a for $\varepsilon=0.4$. The resolution was 50 by 50 grid points for all schemes. For the central difference scheme a term $A \nabla^{2} T$ was added to the right hand side of Eq. (1) in order to reduce non-physical oscillations to a tolerable level. The choice of the diffusivity $A$ is discussed below. At inflow boundaries the constant values $T=0$ and $T=1$, respectively are prescribed, and no boundary conditions are applied at outflow points (in the case of the central difference scheme with diffusion, the diffusive flux is set to zero). The exact steady-state solution for this case has a sharp front at the separation line, with the constant values 0 resp and 1 respectively on both sides. The solutions for the various schemes are displayed in Fig. 2b-d, their mean error is listed in Table 1.

The central differences scheme depends on the choice of diffusion. A value of $A=10^{-3}$ (dimensionless units) which corresponds to a grid Peclet-number $U \Delta x$ / $A=\mathrm{o}(10)$ approximately minimizes the mean error but still exhibits a certain amount of non-physical oscillatory structures (Fig. 2b). A threefold increase in $A$ completely eliminates non-physical values and yields a solution very similar to the upstream case, while the solution for $A=0$ has stronger oscillations and also an increased mean error.

The upstream scheme shows a heavily smoothed front, with a cross-frontal width increasing downstream. Its mean error is somewhat larger than that of the central difference case with optimal choice of diffusion. From scaling considerations the cross-frontal with $\delta$ away from boundaries is given by

$\delta \sim\left(2 A_{\text {eff }} Y * / U\right)^{\frac{1}{2}}$

where $A_{\text {eff }}$ is the effective diffusivity, $U$ the downstream velocity, and $Y_{*}$ a downstream coordinate along the front. For the upstream scheme, the numerical diffusivity is $A_{\text {eff }} \sim \frac{1}{2} U \Delta x$ where $\Delta x$ is the grid resolution, and hence

$\delta \sim\left(\Delta x \cdot Y_{*}\right)^{\frac{1}{2}}$ 

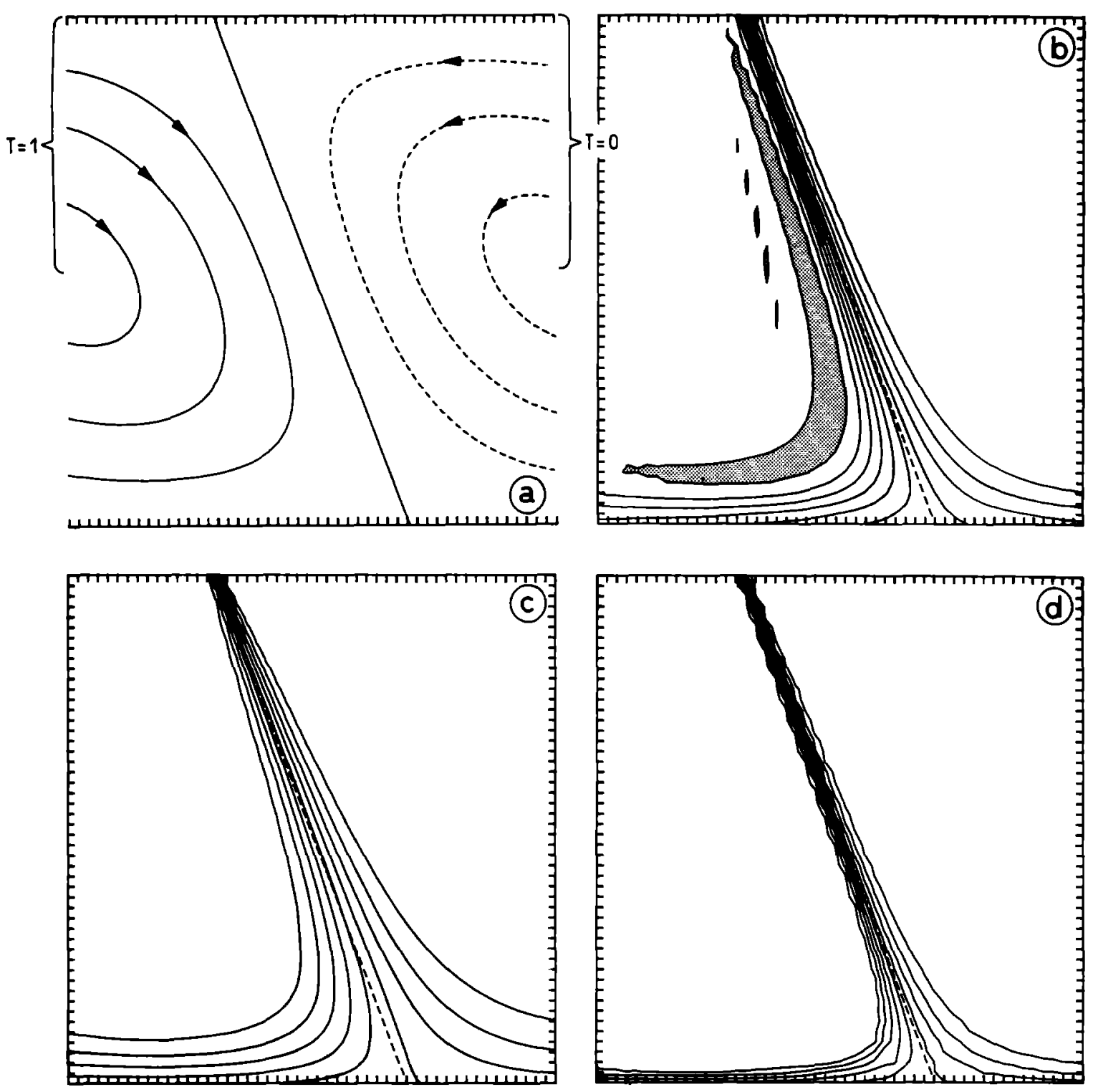

Fig. 2 a-d. Streamlines a and results with b the central differences, c upstream, d FCT numerical advection schemes for a sloping front. Shaded areas in $\mathbf{b}$ indicate that solution exceeds physically possible values

Table 1. Mean error of numerical advection schemes for test cases shown in Fig. 2. The mean error is defined as

$\frac{1}{L^{2}} \int_{0}^{L} \int_{0}^{L}\left|T-T_{\text {exact }}\right| d x d y$

\begin{tabular}{ll}
\hline Scheme & Mean error \\
\hline Exact & 0 \\
\hline Central differences & 0.085 \\
$A=0^{\mathrm{a}}$ & 0.050 \\
$A=10^{-3}$ (Fig. 2b) & 0.069 \\
$A=3 \cdot 10^{-3 \mathrm{a}}$ & 0.076 \\
\hline Upstream (Fig. 2c) & 0.033 \\
\hline FCT (Fig. 2d) &
\end{tabular}

a Not shown
Doubling the resolution (which is achieved at an 8-fold increase in computational expense) hence reduces the cross-frontal width by a factor of $\sqrt{2}$ which is not very effective.

The FCT - scheme is much closer to the exact solution than all other schemes considered here. The crossfrontal width is reduced by almost a factor of $3 \mathrm{com}$ pared to the upstream scheme. A certain amount of waviness in the contours documents the tendency of the FCT-scheme to form small-scale fronts, and is an indication of not strictly monotonous behaviour that may occur in two or more dimenisons (cf. Zalesak 1979).

The front in the preceeding examples was chosen not to be aligned with the grid points, in order to resemble the oceanic situation (here the location of fronts is normally not known a-priori). It is important to note that the above conclusions may be altered in special 

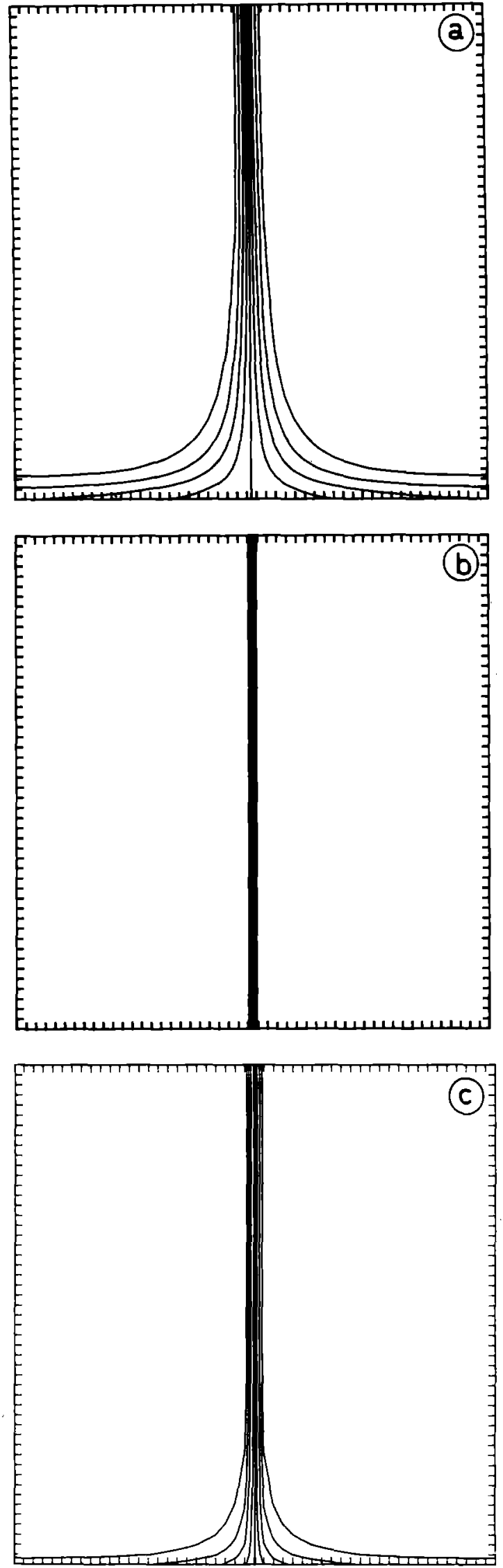

Fig. 3 a-c. Representation of a front aligned with a line of grid points a by the central differences and $\mathbf{b}$, c upstream schemes. a, b are computed with 50 grid points in each direction, in $\mathbf{c} 51$ grid points are used cases where fronts are parallel to the coordinate directions. Figure 3 shows numerical solutions for symmetric streamlines with a vertical separation line $(\varepsilon=0)$. While the central difference solution with explicit diffusion (Fig. 3a) remains qualitatively unchanged compared to Fig. 2b, the upstream solution (Fig. 3b) turns out to be exact. Strictly speaking, this is true only for an even number of grid points, and for a resolution of $51 \times 51$ (Fig. 3c) the solution is exact only in the upper (upstream) half. It is easy to understand this unexpected result by considering the normal velocities along the front. For an even number, the normal velocities vanish between the grid cells adjacent to the front and hence there is no way to transport information across the front. For an odd number of grid points, the normal velocities are opposite and equal (albeit small) on both sides of the innermost grid cells along the front. As long as the normal velocities are directed inside, the front remains sharp as only the innermost grid points are mixed. However, in the lower (downstream) half the signal is transported away from the front so that effective mixing can occur. The FCT is not shown for this case, the results are very similar to those of the upstream scheme. Obviously, the case discussed here is rather special and certainly not typical for an ocean circulation model. However, as will be seen, analogous situations actually can occur in certain regions in oceanic GCMs, and it is important to recognize their origin.

As a preliminary conclusion from these experiments it appears that the FCT scheme performs somewhat better than the other schemes considered here. This is achieved with an approximately threefold increase in computing time compared to the standard schemes. The tendency to enhance fronts on a small scale did not cause a serious problem in the reported example.

As expected, the upstream scheme is very diffusive and a careful choice of explicit diffusion with the central differences scheme yields generally better results which, however, may contain some numerical noise. The noise is due to the same numerical effect responsible for the equatorial salt anomaly in the OGCM mentioned (Fig. 1). An unexpected performance of the upstream scheme was obtained for the special case of a front aligned with the grid, indicating that this scheme may exhibit a pronounced advective character in certain situations.

\section{Experiments with a North Atlantic circulation model}

We have seen that the implicit mixing of certain advection schemes depends strongly on the velocities. This is most pronounced with the upstream scheme but is also the case with the FCT scheme. We must expect analogous behavior in coarse resolution ocean circulation models. The velocity field in an ocean model depends on the individual configuration of the model and we may expect large differences between e.g., a flat-bottom box ocean model and a model with realistic bottom topography and coast line. Altough, for example, the to- 


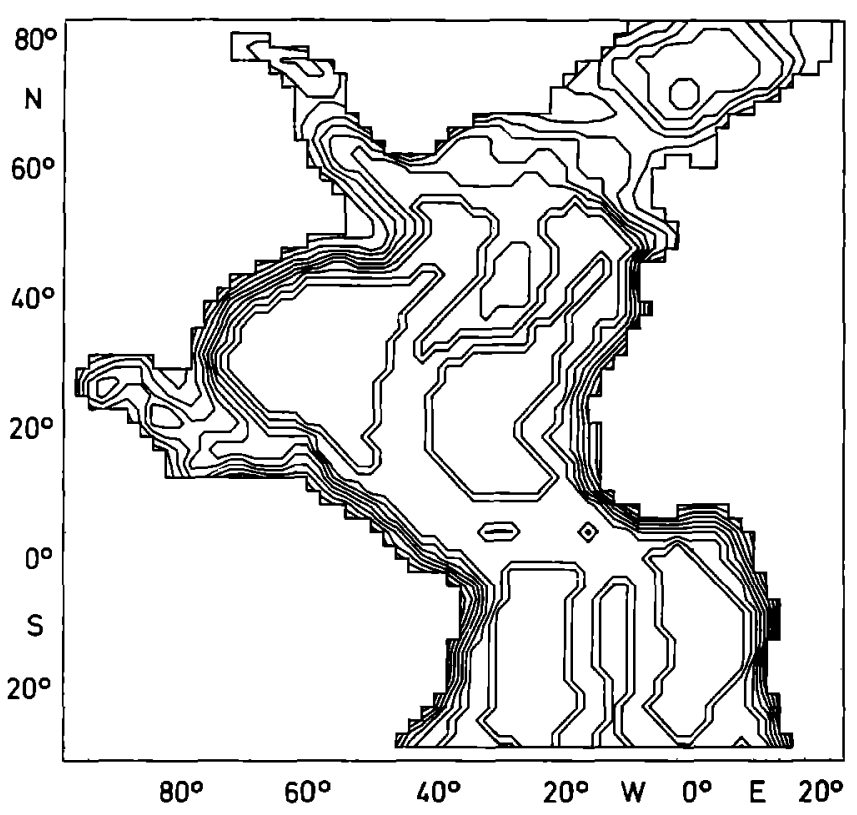

Fig. 4. Domain of the Atlantic model. The bottom topography, after vertical discretization, is included with a contour interval at $500 \mathrm{~m}$

tal overturning rate may be the same in both models, the vertical velocities at individual locations will, in general, be very different due to the kinematic boundary condition at the bottom. While the differences in the vertical velocities may cancel in the zonal average, the vertical mixing will not. Hence, we expect widely different effective vertical mixing rates for both cases. To obtain results that are relevant for typical climate models, it is thus essential, to include such features as bottom topography as well as realistic geometry and atmospheric forcing into the model. For our experiments we selected the Atlantic domain shown in Fig. 4. The boundaries at $30^{\circ} \mathrm{S}$ and $80^{\circ} \mathrm{N}$ are closed walls and the European Mediterranean has been excluded.

The integrations last over 1500 or even 2700 years of simulated time starting from rest and temperatures and salinities taken from the atlas by Levitus (1982). Water masses like the Antarctic intermediate water (AAIW), the Antarctic bottom water (AABW) and the Mediterranean water will not be formed because they are cut off from their source regions by the closed walls. It is, of course, possible to include artifical sources for certain water masses by means of restoring zones in the vicinity of the closed walls (Sarmiento and Bryan 1982; Sarmiento 1986). This may be necessary if one is interested in actual circulation parameters (e.g., heat transport) based on the observed mass field. However, such a procedure obscures the origin of the water masses in the model. With our choice of boundary conditions, the only source for a water mass is the surface and all water masses generated during the integration can be traced back to the surface. As will be shown, the effects of the closed walls on the water mass distribution, although significant, do not account for the main part of the differences between model results and observed distributions. It is obvious, however, that the closed wall at $30^{\circ} \mathrm{S}$ will prevent the establishment of that part of the global thermohaline circulation associated with the "conveyor belt" (Gordon 1986), the flow of near surface waters to high northern latitudes and the corresponding return flow of deep water, which predominantly occurs in the Atlantic Ocean and plays a major role in the global climate.

The numerical models used here are based on the primitive equation model described by Cox (1984) which is a widely distributed code that has been used by many different groups. The model is forced at the surface with the annual mean windstress from Hellerman and Rosenstein (1983) and heat and fresh water fluxes modelled by a Newtonian damping

$Q /\left(\rho_{0} c_{p}\right)=\gamma \Delta z_{1}\left(\theta^{*}-\theta_{1}\right)$

$S_{0}(E-P) / \rho_{0}=\gamma \Delta z_{1}\left(S^{*}-S_{1}\right)$

of the first level temperatures $\theta_{1}$ and salinities $S_{1}$ to prescribed reference distributions $\theta^{*}$ and $S^{*}$ (surface values from Levitus 1982). We have not included the seasonal cycle of $\theta^{*}$ and $S^{*}$ but used the winter distributions which determine the properties of the deep water masses in the ocean. The time scale $1 / \gamma$ for this damping is 30 days and $\Delta z_{1}$ is the thickness of the uppermost gridbox. The vertical grid distances together with the parameters common to all experiments are listed in Table 3. The horizontal resolution is $2^{\circ}$ in both the zonal and meridional directions.

We have carried out three experiments with different advection-diffusion schemes which are summarized in Table 2. The unaltered GFDL-model (Cox 1984) with the central differences scheme for the horizontal and vertical advection of temperature and salinity, serves as a reference experiment. We will refer to this experiment as the standard or CD case. The scalar upstream differences scheme which guarantees the absence of numerical dispersion effects is used in the UPS experiment. Finally, we employ the FCT-scheme for the case with the minimum diffusion that is consistent with the second law of thermodynamics in the FCT experiment. Except for the differences in the advectiondiffusion schemes, the configuration is strictly identical for all experiments. Some technical aspects of the implementation of the upstream and FCT are discussed in the Appendix.

Mixing is parameterized by horizontal and vertical diffusion in the $\mathrm{CD}$ experiment, using constant mixing coefficients $A_{h}=10^{3} \mathrm{~m}^{2} \mathrm{~s}^{-1}$ and $A_{v}=0.65 \cdot 10^{-4} \mathrm{~m}^{2} \mathrm{~s}^{-1}$, respectively. $A_{v}$ was determined experimentally as the smallest value that did not produce the false equatorial salinity anomaly depicted in Fig. 1. It is therefore, the lowest possible constant value that leads to a physically consistent result with this scheme and for this configuration.

The mixing in the UPS experiment is solely provided by the implicit diffusion of the upstream scheme. The effective mixing depends on the absolute value of the velocity component in each coordinate direction (and to a lesser degree on the gradient of the tracer and the shear of the flow). This experiment can be regarded 
Table 2. Advection and mixing schemes used in the Atlantic model

\begin{tabular}{llll}
\hline Experiment & Advection scheme & Explicit mixing & Integration time \\
\hline CD & Central differences & $\begin{array}{l}\text { Horizontal } / \text { vertical } \\
A_{H}=10^{3} \mathrm{~m}^{2} \mathrm{~s}^{-1} \\
A_{v}=0.65 \cdot 10^{-4} \mathrm{~m}^{2} \mathrm{~s}^{-1}\end{array}$ & $2700 \mathrm{yr}$ \\
\hline UPS & Upstream differences & - & $2700 \mathrm{yr}$ \\
\hline FCT & FCT & $\begin{array}{l}\text { iso } / \text { diapycnal } \\
A_{I}=10^{3} \mathrm{~m}^{2} \mathrm{~s}^{-1}, A_{D}=0\end{array}$ & $1500 \mathrm{yr}$ \\
\hline
\end{tabular}

Table 3. Vertical grid and parameters common to all experiments with the Atlantic model

\begin{tabular}{rrrr}
\hline Level & $\Delta z$ & $\begin{array}{l}\text { Depth } \\
\text { of } \\
\text { gridpoint }\end{array}$ & $\begin{array}{l}\text { Depth } \\
\text { of } \\
\text { box bottom }\end{array}$ \\
\hline 1 & 50 & 25.0 & 50 \\
2 & 63 & 81.5 & 113 \\
3 & 92 & 159.0 & 205 \\
4 & 140 & 275.0 & 345 \\
5 & 213 & 451.5 & 558 \\
6 & 313 & 714.5 & 871 \\
7 & 435 & 1088.5 & 1306 \\
8 & 566 & 1589.0 & 1872 \\
9 & 689 & 2216.5 & 2561 \\
10 & 790 & 2956.0 & 3351 \\
11 & 864 & 3763.0 & 4215 \\
12 & 915 & 4672.5 & 5130
\end{tabular}

$\begin{array}{ll}\text { Horizontal resolution: } & \Delta \theta=2^{\circ} \\ & \Delta \lambda=2^{\circ} \\ \text { Horizontal and vertical } & A_{M H}=4 \cdot 10^{4} \mathrm{~m}^{2} \mathrm{~s}^{-1} \\ \text { viscosities: } & A_{M v}=10^{-2} \mathrm{~m}^{2} \mathrm{~s}^{-1} \\ \text { Time constant for the } & \\ \text { Newtonian damping: } & 1 / \mu=30 \mathrm{~d} \\ \text { Time step for } T, S: & \Delta t^{T S}=1 \mathrm{~d} \\ \text { Time step for velocity: } & \Delta t^{\mu v}=2 \mathrm{~h}\end{array}$

as a limiting case of high mixing where the emphasis is on the enforcement of the second law of thermodynamics. Horizontal mixing is expected to be very intense in the western boundary currents and the equatorial region where large velocities prevail. We expect little mixing in regions of slow currents, especially in the eastern parts of the basin. Vertical mixing can be intense in the deep ocean where large vertical velocities are present over variable topography and also in the western boundary layer. The large grid distances in the deeper parts also contribute to enhanced mixing there.

The third experiment represents the opposite extreme to the upstream case. By using the FCT scheme for the horizontal and vertical advection of temperature and salinity, we have attempted to reduce diapycnal mixing as far as possible, in the context of the given coarse resolution grid, without sacrificing basic thermodynamical principles. The implicit diffusion in the FCT algorithm is, by construction in some (subjective) sense, the minimum diffusion that is consistent with the thermodynamical constraint, and we can expect it to be significantly reduced compared to the upstream scheme.
To parameterize adequately the mixing of temperature and salinity due to the (unresolved) eddies, (without introducing additional diapycnal mixing) explicit isopycnal mixing has been added in the FCT case. Isopycnal mixing in a $z$-coordinate model can be described by a mixing tensor as e.g., given by Redi (1982). Some simplifications of the tensor are possible because of the small aspect ratio $\mathrm{H} / \mathrm{L}$ and the smallness of diapycnal compared to isopycnal mixing. Details can be found in the Appendix (see also Gent and McWilliams 1990). Note that the formulation is such that the mixing is always strictly along the local neutral surface.

The values $A_{I}=10^{3} \mathrm{~m}^{2} \mathrm{~s}^{-1}$ and $A_{D}=0$ were chosen for isopycnal and diapycnal mixing coefficients, respectively. In addition to the explicit isopycnal mixing, the FCT-scheme introduces some implicit diffusion in both the horizontal and vertical directions.

The standard and upstream cases were both integrated for 2700 years of simulated time whereas the integration of the FCT case was stopped after 1500 years. Although standard and FCT cases did not reach a complete equilibrium, the trends were well established and could be extrapolated with reasonable confidence. The differences between the models became clear at that point and were not likely to be significantly altered during a continued calculation.

\section{Results}

The adjustment timescales of the ocean to changes in the atmospheric forcing, control the evolution of the full climate system on timescales of decades and centuries. For experiments with coupled ocean - atmosphere models it is, therefore, important to establish those adjustment timescales and their possible dependence on numerical algorithms for the oceanic component. The time evolution of the horizontal averaged temperature from the initial (Levitus) values is an integral measure of the model response to a change in the thermohaline forcing. Density and salinity give no independent information and are not presented here. Features like the decrease of the Mediterranean salinity anomaly can hardly be identified against the overall increase of salinity.

The salient feature of Fig. 5 is a warming trend at all levels on timescales increasing with depth. The adjustment is most rapid in and above the main thermo- 

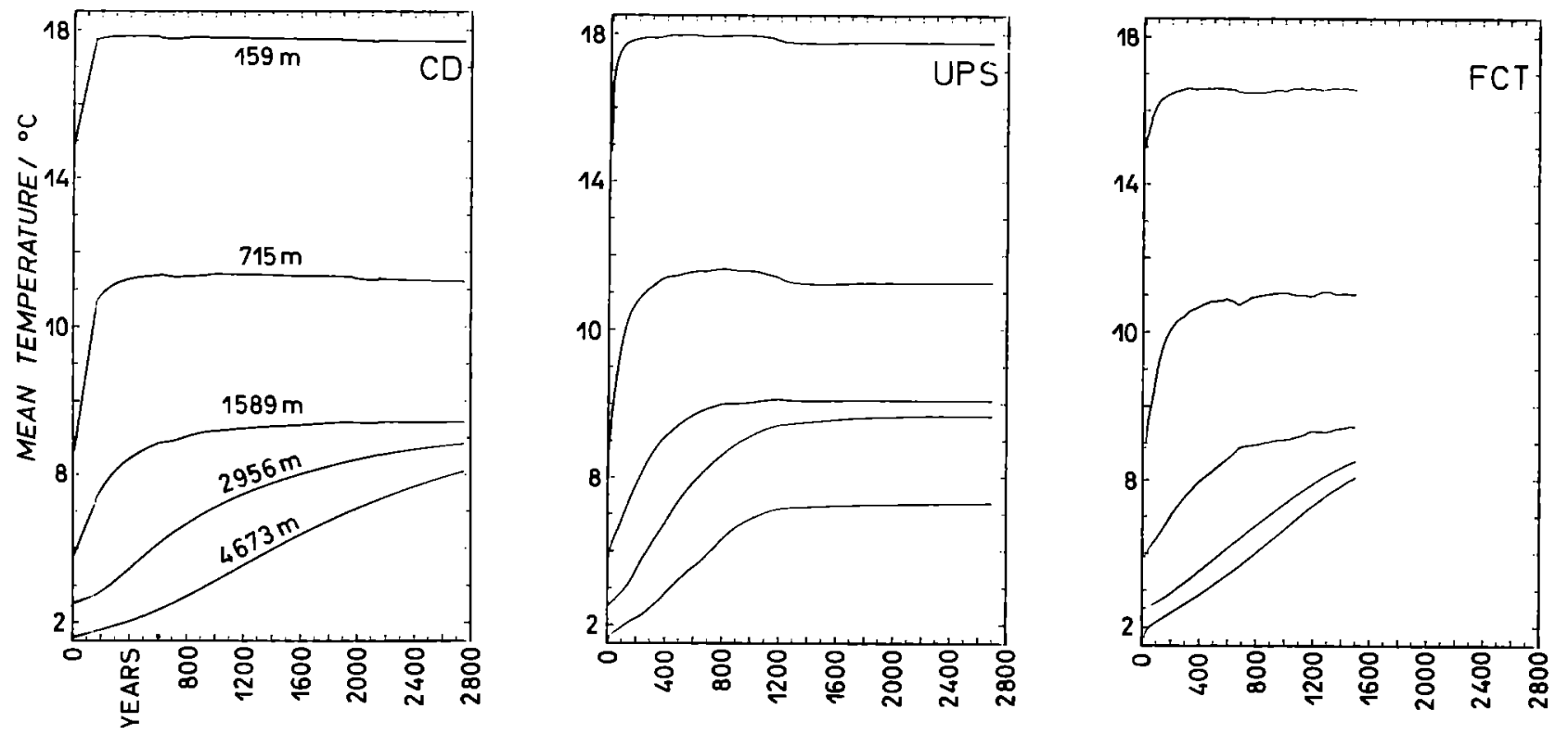

Fig. 5 a-c. Horizontally averaged temperatures as a function of time for the a CD, b UPS and c FCT Atlantic models. Time series for levels $3,6,8,10$, and 12 are plotted

cline where it takes around 100 years to achieve equilibrium. The warming, which in all models occurs mainly at low latitudes, is most pronounced in the standard and UPS models, and somewhat weaker and slower in the FCT model. At the $700 \mathrm{~m}$ level the increase in all models is particularly strong and reaches up to $5 \mathrm{~K}$. At this level, the lack of Antarctic inermediate water (AAIW) formation due to the closed boundary at $30^{\circ} \mathrm{S}$ can be expected to contribute to the warming. The initial salinity anomaly of the AAIW, which covers roughly $45 \%$ of the volume at level 6 (between 558 and 871 meters depth), amounts to -0.3 psu. If this anomaly were removed by isopycnal processes, the temperature would increase by $\Delta T=\beta / \alpha \quad \Delta S \approx 1.8 \mathrm{~K}$ $\left(\beta=0.810^{-3}, \alpha=1.310^{-4} \mathrm{~K}^{-1}\right)$ in the area of the original AAIW and by $0.8 \mathrm{~K}$ in the mean over level 6 . This is less than one fifth of the total increase obtained in that level. Hence we must conclude that the main part of the warming signal has a different origin. It corresponds to a thickening of the main thermocline relative to the initial state. As will be discussed, its most plausible causes are either a too high overall mixing level in these models, or too small upwelling rates, or both.

Even after 2700 years of integration time, the deep levels in the CD-model are not yet in equilibrium, and continue to evolve towards a more or less uniform temperature around $7^{\circ} \mathrm{C}$. A timescale for vertical diffusion is $(1 / 2) z^{2} / A_{v}$, yielding $300(5000)$ years for depths of $1000(4000) \mathrm{m}$ which is not inconsistent with the results for the CD-model. A possible reason for the deep warming is that the formation of North Atlantic deep water by overflow from the Norwegian-Greenland Sea is effectively blocked through lateral diffusion in coarse resolution models. The maximum surface densities south of the overflow region coincide with (winter) temperatures of about $7^{\circ} \mathrm{C}$, and as no interannual fluc- tuations of the surface forcing are considered, we must expect deep temperatures of that magnitude in a steadystate solution. Another possibility, which was pointed out by $\mathrm{K}$. Bryan (personal communication) is that bottom friction is not effective enough to cause a significant ageostrophic downslope flow. The sharp polar front, especially in the upstream and FCT models, indicates that this may be the principal cause, and that lateral diffusion is probably less important.

The closed boundary at $30^{\circ} \mathrm{S}$ which prevents the transport of Antarctic bottom water into the North Atlantic may also contribute to a deep warming in the initial stage of the adjustment. The northward transport of AABW is estimated to be $2.9 \mathrm{~Sv}$ (Roemmich and Wunsch 1985). This implies a cooling of $3 \cdot 10^{-3} \mathrm{~K} / \mathrm{yr}$ for the deepest level in the model assuming a $1 \mathrm{~K}$ temperature difference between the AABW at $30^{\circ} \mathrm{S}$ and the mean temperature over the deepest level. Cutting off the AABW transport by the southern wall leaves the compensating processes that keep the real deep ocean in a nearly steady-state. Warming rates of several $10^{-3}$ $\mathrm{K} / \mathrm{yr}$ are indeed observed in the experiments. However, this warming continues over at least 1000 years, which is far in excess of the time needed to warm the AABW to the initial mean temperature of the deepest level. After a few hundred years of integration, this warming can no longer be attributed to the closing of the southern wall.

The timescales for the upstream-model are significantly shorter, and after little over 1000 years an equilibrium is obtained in all levels. That shorter timescale reflects a larger meridional overturning, especially in the deeper levels (see later). While levels 9 through 11 are homogenized at $7.5^{\circ} \mathrm{C}$, the deepest level remains $2.5^{\circ} \mathrm{C}$ colder. An analysis of the lateral temperature distribution revealed the following reason for this unex- 


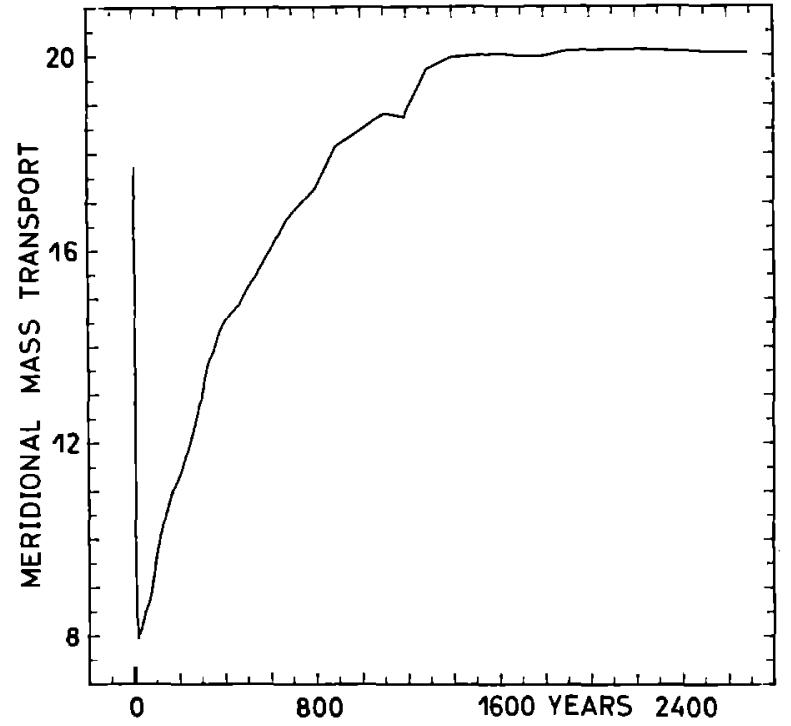

Fig. 6. Maximum meridional mass transport in the deep cell north of $30^{\circ} \mathrm{N}$ for the UPS experiment

pected result. The deepest level is separated into four basins which can only communicate with levels above. Whereas in the North American basin (the best ventilated one) the deepest level indeed has the same average temperature as the level above, e.g., the Brasil basin is at $2.4^{\circ} \mathrm{C}$ which is close to its initial temperature. The vertical velocities in this region are very small, of the order of $10^{-8} \mathrm{~m} / \mathrm{s}$, so that the deepest level is effectively decoupled and remains at its initial temperature (or at the temperature of the time when the vertical velocities become small). This nearly discontinuos behavior is reminiscent of the situation in Fig. 3b which also was caused by small normal velocities in the separation region. The implicit diffusion of the upstream scheme, while generally large, is very small in this region, resulting in a truly advective character of this scheme.

The upstream temperatures in the main thermocline show a small but significant decrease after 1000 years. An indication of this non-monotonic behavior - with a longer timescale and smaller amplitude-can also be seen in the standard model. Apparently that decrease is connected with the evolution of the meridional overturning rate (Fig. 6). Initially the maximum rate plunges within the first decade of integration from 18 to $8 \mathrm{~Sv}$, indicating a sharp reduction in convective mixing and deep water formation at high latitudes. Similarly rapid breakdowns of the thermohaline circulation have been found in models with fixed freshwater flux (e.g., MaierReimer and Mikolajewicz 1989; Marotzke and Willebrand 1991) and also in coupled ocean-atmosphere models (Bryan and Spelman 1985). As the deep levels are gradually warmed, convective mixing becomes more effective, and the total overturning rate increases. That leads to increased upwelling rates in lower latitudes, and hence a decrease in the thermocline temperature. In the other models, this is not seen because the strength of the overturning signal is weaker. Additionally, in the standard model the time-independent cou- pling to the deeper levels through vertical diffusion reduces the influence of the upwelling rate further. Note that the advective time scale $T_{a}=z / W$ is always smaller than the diffusive time scale by a factor of $\Delta z / z$ with the upstream scheme.

The temperatures of the FCT-model at the $150 \mathrm{~m}$ level are significantly lower than in the other two experiments, reflecting a more pronounced gradient in the main thermocline (see Fig. 8 later). At the deepest level the warming after 1500 years is stronger than in the standard version, a consequence of increased mixing necessary to avoid numerical dispersion.

The streamfunction of the meridional mass transport is shown in Fig. 7. The standard case (Fig. 7a) is dominated by a deep cell centered at $1000 \mathrm{~m}$ depth around $50^{\circ} \mathrm{N}$, reaching a maximum value of $14 \mathrm{~Sv}$. Sinking is confined to a small region near $60^{\circ} \mathrm{N}$, mainly the Irminger Sea, and only 2-3 Sv originate from exchange with the Norwegian-Greenland Sea. Very little transport occurs below $2500 \mathrm{~m}$. Near the surface three cells can be identified, their upper portions obviously result from Ekman transport. The strongest cell reaches $12 \mathrm{~Sv}$ between $10^{\circ} \mathrm{N}$ and $20^{\circ} \mathrm{N}$. The equatorial upwelling mainly occurs from shallow depths, but approximately $4 \mathrm{~Sv}$ are connected to the deep cell. Although the southern boundary is closed, there still is a cross-equatorial northward near-surface transport of 4 Sv.

While the upper ocean pattern in the upstream model (Fig. 7b) is more or less identical to the standard case, the deep overturning cell has a maximum transport of nearly $20 \mathrm{~Sv}$, a $40 \%$ increase over the standard model. The difference is most pronounced between 2000 and $3000 \mathrm{~m}$ north of $40^{\circ} \mathrm{N}$, as seen from the difference map (Fig. 7d). The ventilation of the deepest levels in mid-latitudes is strongly enhanced compared to the standard case, causing the much faster adjustment of the upstream model. In contrast, the FCT model (Fig. 7c) has a transport maximum of only $11 \mathrm{~Sv}$, and the deep circulation is weaker than in both other models. The FCT model shows almost no low latitude upwelling from deeper levels into the main thermocline.

The temperature section at $30^{\circ} \mathrm{W}$ (Fig. 8) gives an impression of how the different models are able to simulate the main thermocline structure. The standard and the upstream models (Fig. 8a, b) are nearly identical. Both fail to reproduce the observed sharp gradients (Fig. 8d), especially in the equatorial region. For the standard version, this problem has been noticed long ago (e.g., Bryan et al. 1975) and attributed to too much vertical diffusion and the weakness of the thermohaline circulation. The upstream scheme does not give any improvement which one might have expected in analogy to Fig. 3b. The reason is a strong exchange across the main thermocline levels in some parts of the basin. While e.g., the net upwelling into level 4 between $20^{\circ} \mathrm{S}$ and $20^{\circ} \mathrm{N}$ amounts to $7 \mathrm{~Sv}$, this number consists of 15 $\mathrm{Sv}$ total upwelling accompanied by $8 \mathrm{~Sv}$ downwelling. Implicit diffusion through lateral advection then results in a broad thermocline structure. 

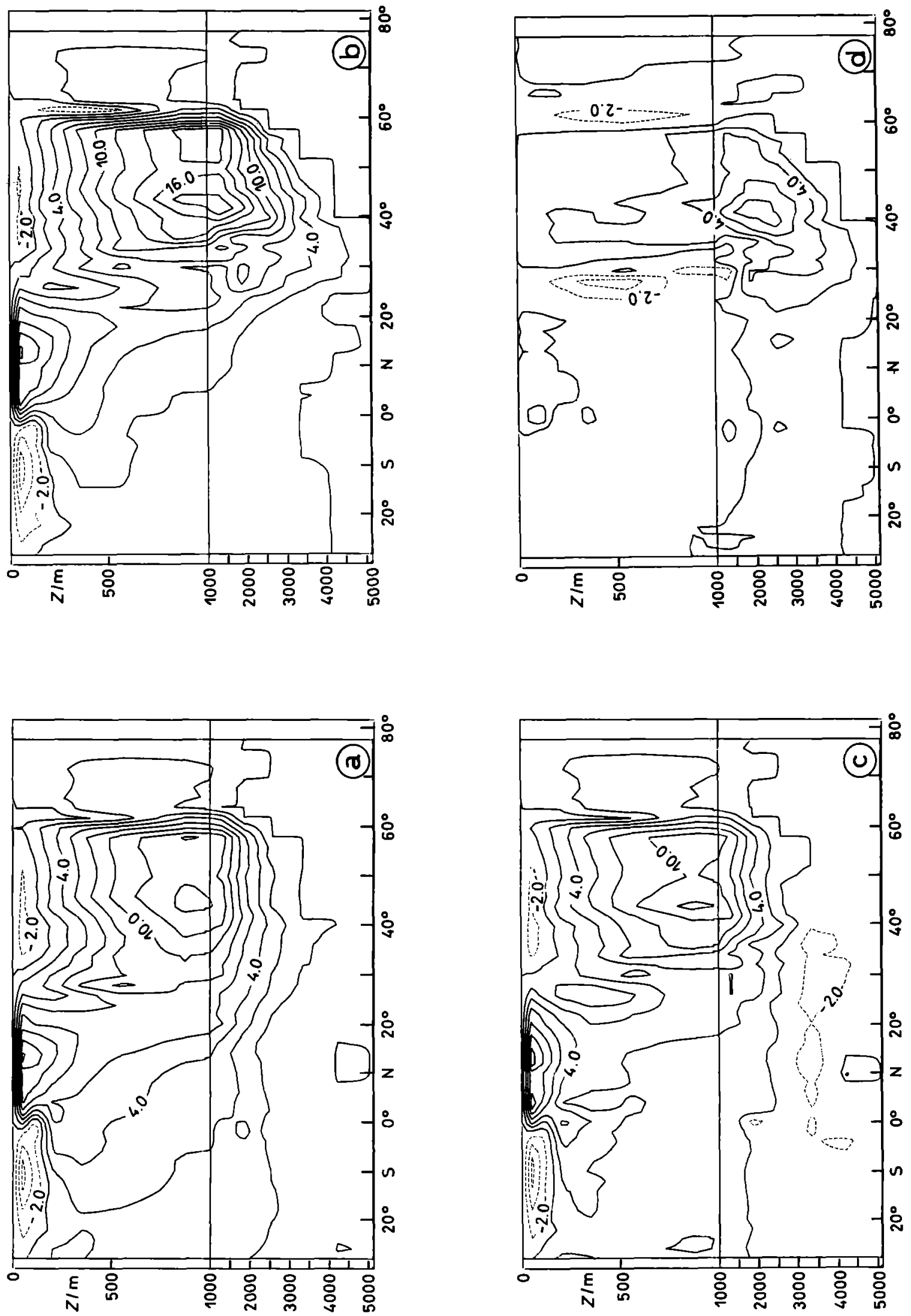

Fig. 7 a-d. Meridional mass transport for the a CD, b UPS and $\mathbf{c}$ FCT experiments. The difference between UPS and CD is plotted in d. The contour interval is $2 \cdot 10^{6} \mathrm{~m}^{3} / \mathrm{s}$ 

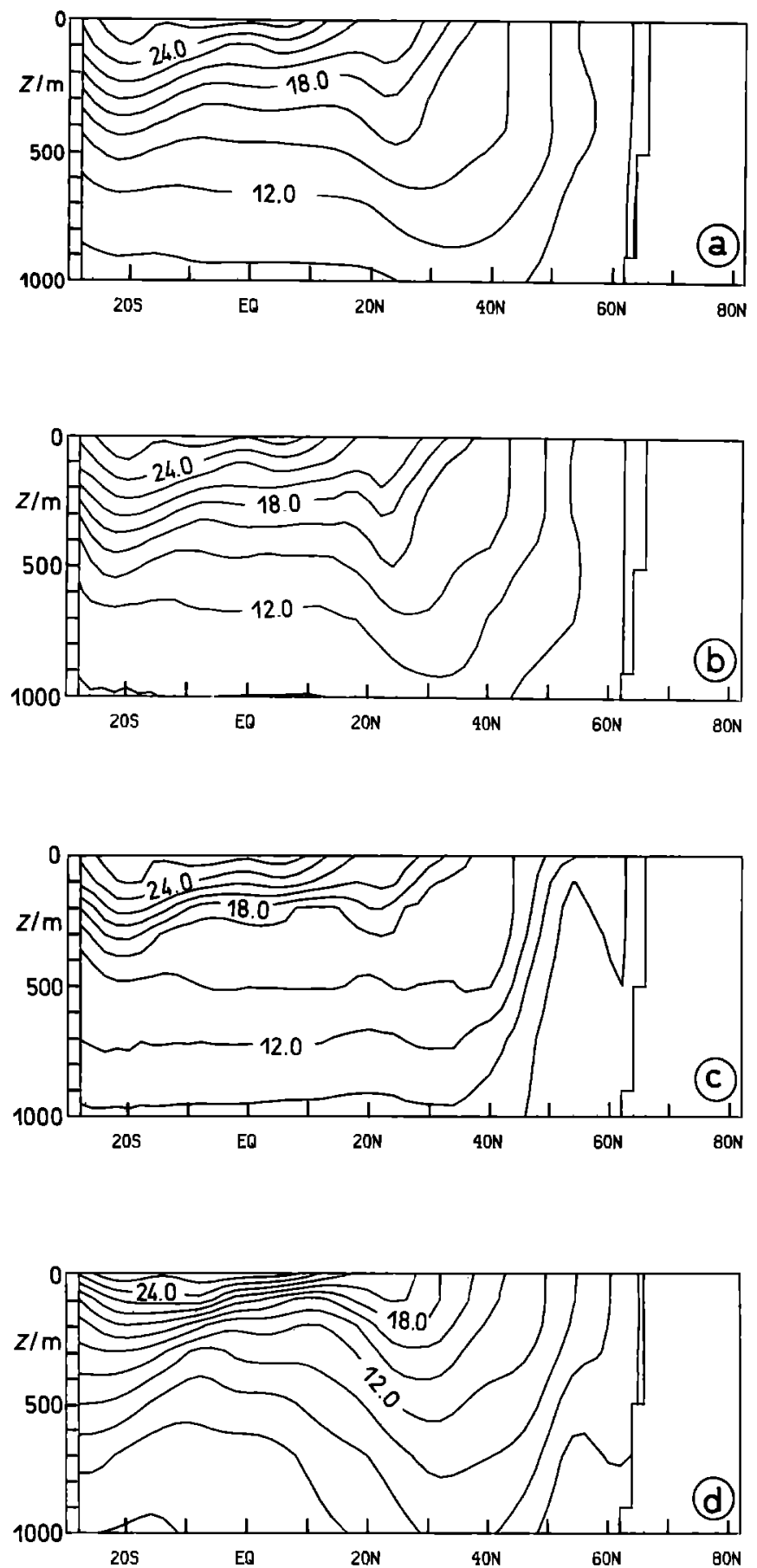

Fig. 8 a-c. Temperature section along $30^{\circ} \mathrm{W}$ for the a CD, b UPS, and c FCT experiments. The contour interval is $2 \mathrm{~K}$

Horizontal diffusion of temperature differences that are due to upwelling and downwelling in different regions also contributes to the broad thermocline structure in the standard model. In contrast, the isopycnal mixing in the FCT model (Fig. 8c) does not wipe out these differences. The equatorial thermocline in the FCT model is shallower, and the vertical gradients seem much more realistic. The subarctic front is significantly sharper than in the other models. However, the maximum in thermocline depth near $30^{\circ} \mathrm{N}$ is not well reproduced, and the isotherms do not rise towards the equator.

F. Bryan (1987) has demonstrated in a series of experiments that both the strength of the meridional overturning and the thermocline thickness are closely related to the vertical mixing coefficient $A_{v}$. He found that both increase with $A_{v}^{1 / 3}$, a rule which, for the thermocline thickness, can also be deduced from scaling arguments, whereas for the overturning transport, scaling would suggest a $A_{v}^{2 / 3}$ law.

An integral measure for the thermocline depth is

$d=\int_{-H}^{0} \frac{\left(T-T_{s}\right) d z}{\left(T_{s}-T_{H}\right)}$

where $T_{s}$ and $T_{H}$ are the temperatures at the surface and at depth $z=-H$ respectively. Values for the mean profiles between $10^{\circ} \mathrm{S}$ and $10^{\circ} \mathrm{N}$ are presented in table 4 for $H=500 \mathrm{~m}$. The effective mixing rates, according to the $A^{1 / 3}$ relation, indicate that the overall level of diapycnal mixing in the equatorial region is somewhat stronger in the upstream model than in the standard case. The mixing is considerably reduced in the FCT model. The product of $\boldsymbol{d}$ and a mean upwelling velocity gives an estimate of the vertical diffusivity, that does not rely on a specific model assumption, unlike the scaling mentioned previously. The results (Table 4) are, however, consistent and lead to the same conclusions. The horizontal average over the limited equatorial domain does not take into account the effect of the lateral fluxes into the domain. Horizontal fluxes vanish in the average over the total area. A more appropriate measure for the overall effective vertical mixing is therefore given by

$\left\langle A_{v}\right\rangle=\left\langle F^{D}\right\rangle /\left\langle T_{z}\right\rangle$

where $(\ldots)$ denotes the horizontal average and $F^{D}$ is the diffusive vertical flux of temperature. In the standard case $\left\langle A_{v}\right\rangle$ is equal to the mixing coefficient $A_{v}=0.65 \cdot 10^{-4} \mathrm{~m}^{2} \mathrm{~s}^{-1}$. Vertical distributions of $\left\langle A_{v}\right\rangle$ for the UPS and FCT models are displayed in Fig. 9. In the UPS model $\left\langle A_{v}\right\rangle$ increases linearly from 0.4 to a maximum of $4.5 \cdot 10^{-4} \mathrm{~m}^{2} \mathrm{~s}^{-1}$ near $3000 \mathrm{~m}$ depth. In the deep ocean $\left\langle A_{v}\right\rangle$ almost vanishes. The effective mixing is reduced with the FCT scheme except for the deepest level that is partly decoupled from the rest of the basin in the UPS model. The mixing rates increase with depth and exceed the $0.65 \cdot 10^{-4} \mathrm{~m}^{2} \mathrm{~s}^{-1}$ employed for the CD model below $1000 \mathrm{~m}$ depth. These large mixing rates in deeper layers, indicate that $0.65 \cdot 10^{-4}$ is still too small to preserve monotonicity with the central differences scheme. The small mixing rates in the upper layers, seem to be of greater importance than the large ones in deeper levels, with respect to meridional masstransport and thermocline depth.

The meridional heat transport for all models is shown in Fig. 10. The standard run is characterized by a strong heat gain in the equatorial zone, reaching a maximum of $0.55 \mathrm{PW}$ at $8^{\circ} \mathrm{N}$ and falling off more or less uniformly towards high latitudes. Following Bryan's (1962) terminology, most of the total heat trans- 


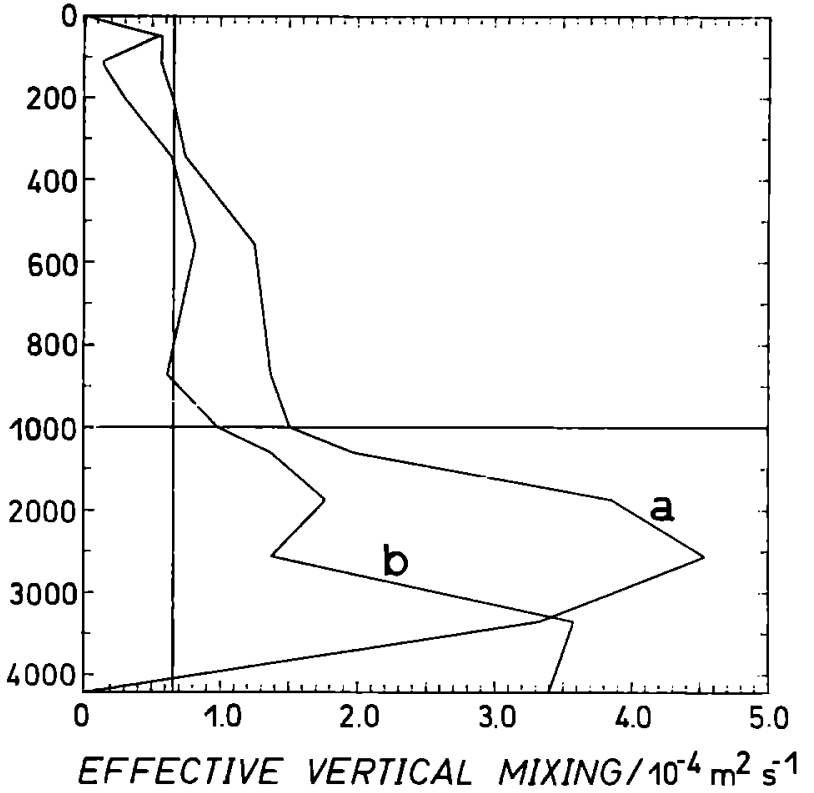

Fig. 9a, b. Effective vertical mixing rate $\left\langle A_{v}\right\rangle$ (see text) in the a UPS and b FCT experiments. The constant mixing rate of the CD experiment is indicated by the solid line

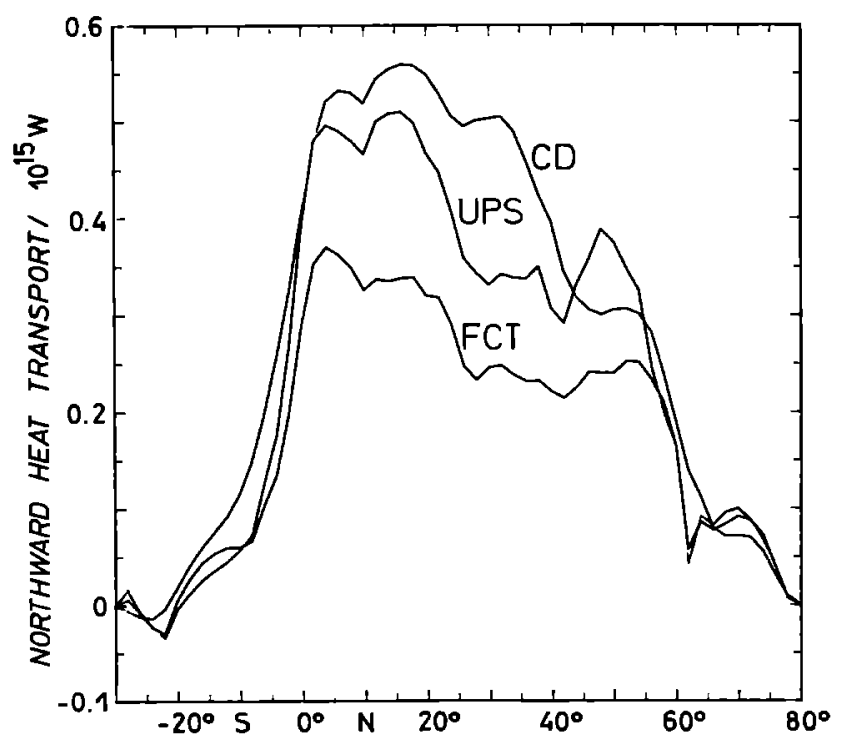

Fig. 10. Northward heat transport in PW for the CD, UPS, and FCT experiments

port is due to the overturning component. Only northward of $40^{\circ} \mathrm{N}$ the gyre component becomes dominant. The upstream heat transport is slightly smaller at low latitudes, but is larger north of $40^{\circ} \mathrm{N}$. The secondary maximum near $50^{\circ} \mathrm{N}$ is caused by a maximum in the gyre transport which is more pronounced than in the standard case.

The increase in northward heat transport north of $40^{\circ} \mathrm{N}$ corresponds to a heat gain of the ocean through the surface. That heat gain originates from the Newfoundland Basin and has already been noticed by Sar-
Table 4. Characteristic results from the experiment with the Atlantic model. See text for the definition of the thermocline depth $d$ and the effective vertical diffusion $A_{\text {velf. }} . \bar{w}$ is the average upwelling velocity at $113 \mathrm{~m}$ depth between $10^{\circ} \mathrm{S}$ and $10^{\circ} \mathrm{N}$. The "diagnostic" case was computed from the UPS model after 2 months of integration

\begin{tabular}{|c|c|c|c|c|}
\hline & CD & UPS & FCT & $\begin{array}{l}\text { Diagnostic } \\
\text { case }\end{array}$ \\
\hline Thermocline depth $\mathrm{d} / \mathrm{m}$ & 173 & 186 & 131 & 149 \\
\hline$A_{v e r[} /\left(10^{-4} \mathrm{~m}^{2} \mathrm{~s}^{-1}\right)$ & 0.65 & 0.81 & 0.28 & 0.42 \\
\hline$d \cdot \bar{w} /\left(10^{-4} \mathrm{~m}^{2} \mathrm{~s}^{-1}\right)$ & 0.95 & 1.17 & 0.31 & 0.76 \\
\hline $\begin{array}{l}\Psi_{\max } / \mathrm{Sv} \\
\text { (subtropical gyre) }\end{array}$ & 41 & 50 & 40 & 58 \\
\hline $\begin{array}{l}\Psi_{\max } / \mathrm{Sv} \\
\text { (subpolar gyre) }\end{array}$ & 28 & 33 & 26 & 34 \\
\hline $\begin{array}{l}\text { Maximum meridional } \\
\text { mass transport/Sv }\end{array}$ & 14.7 & 20.0 & 12.8 & 19.9 \\
\hline $\begin{array}{l}\text { Maximum northward } \\
\text { heat transport/PW }\end{array}$ & 0.56 & 0.51 & 0.37 & 1.21 \\
\hline
\end{tabular}

miento (1986). A cold SST anomaly due to an eastward flowing Labrador Current and southward Ekman transport, forces the model ocean to take up heat through the restoring boundary condition, in a region where observations indicate strong ocean heat-losses. The UPS model shows a larger heat gain due to its more intense subarctic gyre (Table 4) which also penetrates farther south than in the CD model.

The FCT model has a significantly reduced heat transport, gaining a maximum of $0.35 \mathrm{PW}$. This difference is caused by a much weaker overturning component, whereas the gyre transport alone slightly exceeds the other models.

It may be instructive to compare the heat transport with Sarmiento's (1986) results. There 0.48 PW are gained in the restoring zone near the southern boundary, simulating the heat transport across $30^{\circ} \mathrm{S}$. The heat transport stays almost constant until the equator is reached, where roughly $0.5 \mathrm{PW}$ are gained by Sarmiento's model. This is almost the same value as in the CD and UPS models described here. The equatorial heat gain comes out again as a very robust feature of different models. It is obviously determined by the Ekman transports which are given by the prescribed forcing. The deep upwelling gives only a minor contribution to the air-sea temperature difference. Sarmiento's model again shows almost constant heat transport north of the equator until the total heat is given up within the northern restoring zone. The models considered here show a much stronger release of heat north of the equator which seems to compare better with observations (e.g., Isemer and Hasse 1987). 


\section{Discussion}

We have investigated the sensitivity of coarse-resolution ocean circulation models on the choice of the numerical advection scheme, and on the formulation of the mixing parametrization. The principal conclusion of this study is that characteristic properties relevant for climate response experiments such as e.g., meridional overturning and heat transport, thermocline thickness and horizontal gyre transport plus the timescale for the adjustment to thermohaline forcing, depend not only on explicit mixing parameters but also on implicit parameters hidden in the choice of the numerical scheme.

Three cases (standard, UPS and FCT) have been considered. The standard case uses the traditional central differences algorithm with explicit vertical and horizontal diffusion. The (constant) values for the diffusion parameters are rather large, leading e.g., to a main thermocline structure which is too broad compared to observations. Nevertheless, temperature and salinity distributions still show signs of non-physical oscillations. In the deep ocean temperature and salinity budgets are dominated by vertical diffusion, leading to rather long adjustment timescales of several thousand years.

The choice of the vertical diffusion coefficient was not primarily based on physical considerations. To avoid numerical dispersion effects like the equatorial salinity maximum shown in Fig. 1 we were forced to use a vertical diffusivity of $0.65 \cdot 10^{-4} \mathrm{~m}^{2} \mathrm{~s}^{-1}$. Central differences models with vertical resolution similar to our experiments have been described in the literature, using much smaller vertical mixing rates. A recent example can be found in Bryan (1987) where the vertical diffusivity is reduced to $10^{-5} \mathrm{~m}^{2} \mathrm{~s}^{-1}$ for one experiment with a 12-level flat-bottom box ocean model. We do not imply that those results are necessarily contaminated by numerical dispersion effects as the occurrance of such effects depends very much on the individual configuration. The vertical velocities in flat-bottom cases are, in general, smaller and with that the sensitivity to numerical dispersion is diminished. However, a recent study with a flat-bottom 12-level model has identified severe numerical errors leading to a false equatorial cell (Weaver and Sarachik 1990). The surface boundary values, for temperature and salinity applied in Bryan's calculation, also do not contain such extremes as our boundary values in the Guinea basin, which are taken from Levitus (1982) without further modifications. The very low surface values in our case give rise to a sharp front below the surface which is especially susceptible to numerical dispersion effects.

However, in many cases dispersive effects are less dramatic than those shown in Fig. 1 and may remain unnoticed. The temperature section corresponding to Fig. 1 for example shows only a spreading of the isotherms in the depth range of the salinity anomaly and lacks the conspicuous structure visible in the salinity field. A temperature anomaly is nevertheless present, generated similarily to the salinity anomaly at the same location. In this particular case the anomaly does not destabilize the density stratification. However, dispersive ripples develop independently in the different advected quantities, and in general there will be no compensation in the density field. Due to the convective adjustment this will often lead to erroneous vertical fluxes of heat and salinity and an artificially increased vertical mixing which may be difficult to detect.

The upstream model overall is by far the most diffusive one of those considered here. Its implicit diffusion is always sufficient to suppress numerical dispersion effects. The spatial distribution of implicit mixing is nonuniform. Compared to the standard case, effective mixing is comparable in the main thermocline while much larger values prevail in the deep ocean. Meridional overturning is significantly stronger and extends to larger depths. The amount of $20 \mathrm{~Sv}$ compares favorably with estimates from the observed mass and tracer fields (e.g., Roemmich and Wunsch 1985) as well as with the result of our 'diagnostic' case. The horizontal transport increases by approximately $20 \%$ in both subtropical and subpolar gyres. This increase is related to the more vigorous deep flow in the upstream case. Regionally (e.g., at the polar front and in the Brasil Basin) advective characteristics in the $T-S$ distributions are more visible than in the standard case.

The FCT-model with isopycnal mixing parametrization was constructed to minimize the effective diapycnal mixing while satisfying the thermodynamical constraints. The effective mixing is particularly small in the thermocline, and a significant reduction in thermocline thickness which is much closer to the observations has been achieved in this way. Below $1000 \mathrm{~m}$ the diapycnal mixing of the FCT-model exceeds that of the standard run, and we must conclude that for the present resolution a value of $0.65 \cdot 10^{-4}$ is too small to avoid numerical dispersion effects everywhere. Apparently, mixing coefficients increasing below the main thermocline (which actually may be motivated from physical considerations, see Gargett 1984) would be better suited for this purpose.

The most striking discrepancy between model results and observations is the warming trend in all models (Fig. 5) leading to higher model temperatures in the deep ocean. We have ruled out the limited computational domain as a principal cause for this discrepancy. The analysis of the model results in terms of the effective vertical diffusion showed that the warming mechanism is different among the models. For the standard case we found a purely diffusive adjustment of the deep ocean towards the temperature at the base of the deepest level which is directly ventilated by the meridional overturning motion. This is not primarily due to an overly large mixing coefficient but to the virtually complete lack of motion in these deep layers. The deepreaching overturning in the upstream case provides for an advectively dominated regime in spite of the large mixing rates in the deep levels. However, the North Atlantic deep water distributed by the current system has the wrong properties. The relatively large vertical mixing rates below $1000 \mathrm{~m}$ in the FCT case (Fig. 9) indicate 
large vertical velocities between those levels. This does not appear in the meridional mass-transport (Fig. 7) because the motion is on small horizontal and vertical scales and tends to cancel in the zonal integral. Small scale motion is ineffective in changing the large scale tracer distributions. The associated implicit mixing of the FCT scheme can nevertheless be an important process, as seen from the fast adjustment of the deepest levels compared to the standard case in the absence of a strong large scale overturning motion.

Due to the small effective mixing rates in the upper $500 \mathrm{~m}$ only the FCT model succeeds in simulating a realistic equatorial thermocline. The relation between thermocline thickness and effective vertical mixing rates in the upper levels (Table 4) is consistent with the results of the series of experiments with different, but constant, vertical mixing coefficients conducted by Bryan (1987). The reduction in meridional overturning and heat transport in the FCT case compared to the standard and upstream cases is also consistent with the lower mixing rates in the upper $500 \mathrm{~m}$. The differences in the effective deep mixing rates, on the other hand, are obviously inconsistent with Bryan's results. The standard case and the upstream case, which have completely different mixing rates in the deep levels, yield large transports, while the FCT case with large mixing rates, yields only very small meridional transports. This indicates that for the above properties the mixing rates at great depths are less important than the rather subtle differences in the upper thermocline.

How can one choose between models which use different numerical algorithms or different subgridscale parametrizations? As long as the physical (and mathematical) basis of different schemes seems more or less equally justified, the only way to tell which one is "best" is by them comparing to oceanic observations. We have been somewhat restricted in performing a systematic comparison with the observed mass field in the Atlantic. For such a purpose, models on a global domain, which are not influenced by artificial boundaries, would clearly be more appropriate, and other important factors such as e.g., seasonal and interannual variability in the surface forcing, would have to be included. Also, some more experience with different parameter settings is necessary before a useful comparison to data can be made.

The result of a systematic comparison will, of course, depend on which aspect of the observations is emphasized most or, in the jargon of inverse modeling, which "cost function" is chosen. For models of water mass distribution, we believe that the equivalent of negative concentrations (i.e., unmixing) should have a high penalty because it violates a basic thermodynamic principle. If this premise is accepted, then the upstream scheme seems preferable to the standard case for coarse-resolution models, despite its somewhat lower overall accuracy (and its lower popularity among ocean modellers). The FCT-scheme offers similar advantages as the upstream scheme at a somewhat higher accuracy. Disadvantages of the FCT scheme are the subjective choice of the limiting process, a rather complicated al- gorithm, and the increased computational effort (approximately a factor of 2 in our GCM calculations).

It is apparent from Fig. 9 that, for a configuration with 12 vertical levels, none of the schemes considered here can allow for mixing rates as small as $10^{-5} \mathrm{~m}^{2} / \mathrm{s}$ which are suggested from microstructure observations in the main ocean thermocline (Gregg 1987). A significant increase in vertical resolution would be necessary to achieve this purpose. Models with much higher vertical resolution are, of course, less sensitive to implicit mixing due to numerical algorithms, and the secondorder accuracy of the central difference scheme ultimately becomes very attractive. As the computational costs increase only linearly with vertical resolution, increasing the vertical resolution seems a sensible and feasible approach for ocean circulation models which are used for climate studies requiring long-term integrations.

Acknowledgements. We are indebted to the late Michael Cox for providing the code for the numerical model used in this study, and to Kirk Bryan for helpful comments on an earlier version of this manuscript. Support by the Nationales Klimaforschungsprogramm under contract KF 21218 - TV 4 is gratefully acknowledged.

\section{Appendix 1}

\section{Implementation of upstream and FCT schemes}

Because of the diffusive character of the upstream discretization a forward timestep

$T^{n+1}=T^{n}-\Delta t\left\{\nabla \cdot\left(u^{n} T^{n}\right)+R\left(T^{n}\right)\right\}$

would normally be employed where $R$ contains the contributions other than advection to the tracer balance. However, this discretization turned out to be unstable in the ocean model. The most likely cause is a nonlinear instability of the full system containing the momentum, temperature and salinity balances.

There are several ways to suppress that instability. Here we chose

$T^{n+1}=T^{n-1}-2 \Delta t\left\{\nabla \cdot\left(u^{n} T^{n-1}\right)+R\left(T^{n-1}\right)\right\}$

over $2 \Delta t$ which is stable and also facilitates the combination of fluxes from the central difference scheme with leapfrog timestepping, with fluxes from the upstream scheme in the FCT case.

The implementation of the FCT scheme follows the outline given by Zalesak (1978). The limit for the antidiffusive fluxes are chosen to assure that the new solution $T^{n+1}$ lies within a certain range $T_{\min }$ and $T_{\max }$. These limits for $T^{n+1}$ at a certain grid point $(i, j, k)$ are given by

$$
\begin{aligned}
T_{i j k}^{\min / \max }= & \min _{\max }\left\{T_{i j k}^{*}, T_{i j k}^{n}, \frac{1}{2}\left(T_{i j k}^{n}+T_{i+l j k}^{n}\right),\right. \\
& \frac{1}{2}\left(T_{i j k}^{n}+T_{i-l j k}^{n}\right), \frac{1}{2}\left(T_{i j k}^{n}+T_{i j+l k}^{n}\right), \\
& \frac{1}{2}\left(T_{i j k}^{n}+T_{i j-l k}^{n}\right), \frac{1}{2}\left(T_{i j k}^{n}+T_{i j k+1}^{n}\right), \\
& \left.\frac{1}{2}\left(T_{i j k}^{n}+T_{i j k-1}^{n}\right)\right\} .
\end{aligned}
$$


This is a slight modification of Zalesak's scheme. The mean of neighboring $T^{n}$ (the solution of the preceding timestep) replaces the upstream solution $T^{*}$ at time $n+1$. This modification allows a more simple and efficient coding and has no significant influence on the results. The solution of the advective step cannot be outside the range $T^{\mathrm{min} / \max }$. This does not assure, however, the strict monotonicity of the advected quantity, and the results may still show unrealistic wavelike behavior.

It is possible that the range of allowed values is given by the variation of $T$ in only one direction, whereas the largest antidiffusive flux occurs in a direction nearly perpendicular to the gradient of $T$. This is indeed often the case in the ocean model where the transports are predominantly horizontal whereas the gradient is almost vertical. In order to overcome this problem, we have employed 1-dimensional prelimiters as suggested by Zalesak. The allowed range $T 1^{\mathrm{min} / \mathrm{max}}$ for the 1-dimensional FCT-steps is determined for each coordinate direction in analogy to (A.3).

The use of 1-dimensional prelimiters is not the only way to achieve sufficiently monotonic distributions. We have conducted experiments with a 2-dimensional FCT for the horizontal directions and a 1-dimensional scheme for the vertical to prelimit the antidiffusive fluxes. This introduces some more noise but on the other hand is less diffusive than three 1-dimensional limiters.

\section{Appendix 2}

\section{Remarks on the isopycnal mixing formulation}

1. We combined the FCT advection scheme only with isopycnal mixing because the large diffusion in the horizontal present in the upstream experiment would have masked any additional isopycnal mixing. Temperature and salinity as well as other tracers are found to be almost homogeneously distributed on density surfaces in large areas of the model. This is the result of the advectively dominated tracer balances which prevail in the upper thermocline and is even the case when no isopycnal mixing is acting. Isopycnal mixing by definition has no effect on tracers that are homogenous on density surfaces. As noted by Cox (1987 unpublished manuscript), the numerical consequence is that isopycnal mixing, unlike horizontal mixing, does not contribute to the suppression of numerical dispersion in these cases, and hence must be accompanied by either a fluxlimiting scheme as in our FCT-experiment or additional horizontal mixing.

2. Large vertical fluxes of tracers associated with isopycnal mixing along steeply sloping density surface can cause numerical instability due to violation of the CFLcriterion. It is therefore necessary to limit the vertical fluxes depending on the slope of the isopycnals. This is done without introducing any diapycnal mixing by reducing the isopycnal mixing coefficient while keeping the isopycnal/diapycnal orientation of the mixing tensor. The diffusion tensor then takes the form (cf. Gent and McWilliams 1990)

$$
\boldsymbol{A}=A_{I} / S^{2}\left(\begin{array}{lll}
\rho_{z}^{2} & 0 & -\rho_{x} \rho_{z} \\
0 & \rho_{z}^{2} & -\rho_{y} \rho_{z} \\
-\rho_{x} \rho_{z} & -\rho_{y} \rho_{z} & A_{D} S^{2} / A_{I}+\rho_{x}^{2}+\rho_{y}^{2}
\end{array}\right)
$$

and has the same principal axes (but slightly different eigenvalues) as the full tensor. Except for the off-diagonal elements $-\rho_{x} \rho_{y} / \rho_{z}^{2}$, which we neglected for the sake of consistency, these are the same approximations as given by $\operatorname{Cox}$ (1987). $A_{I}$ and $A_{D}$ denote isopycnal and diapycnal mixing, respectively. The parameter $S$ serves to limit the mixing along a steep isopycnal, and is given by

$$
S=\min \left\{\rho_{z},-\left(\rho_{x}^{2}+\rho_{y}^{2}\right)^{\frac{1}{2}} / \tan \alpha\right\} .
$$

Here $\alpha$ is the inclination of the isopycnal surface against the horizontal which has to be chosen according to the stability criterion given by Cox (1987):

$\alpha_{\max }=\min \left(\frac{\Delta z}{\left(4 A_{I} \Delta t\right)^{1 / 2}}, \frac{\Delta x \Delta z}{4 A_{I} \Delta t}, \frac{\Delta y \Delta z}{4 A_{I} \Delta t}\right)$

Note that $\rho$ in the above expressions denotes the potential density referred to the actual level of computation so that isopycnal mixing is strictly along the local neutral surface.

3. During initial experiments with the isopycnal mixing scheme, there occured temperatures and salinities far beyond the physically possible range. These anomalies were confined to grid points which were topographically isolated from advection. Although all surrounding grid points showed higher temperatures and salinities, their temperature and salinity fell continuously. The cause of this unexpected behavior was a negative diffusion effect due to the discrete nature of the model. At boundaries where fluxes and flux divergences have to be computed by one-sided rather than central differences, the discrete form of the isopycnal mixing term can lead to "unmixing" despite a positive value of the coefficient $A_{I}$. Ironically the mixing formulation in this case causes a violation of the second law of thermodynamics which is exactly what we have tried to avoid by careful choice of the advection scheme.

The problem results from the description of isopycnal mixing in the framework of a $z$-coordinate model. It will not appear in a model based on isopycnal coordinates. We have solved it by introducing boundary layers one grid distance wide along the whole boundary of our domain (except for the surface) where the diffusion is horizontal instead of isopycnal and hence contains a diapycnal component. Although not motivated from physical considerations, this may also be physically appropriate because of the larger energies available along the boundaries to mix properties across isopycnals. This is somewhat similar to the approach of Cox (1987) who retained a certain amount of horizontal mixing. While we restrict this mixing to a boundary layer, Cox, however, has also applied it in the interior. 


\section{References}

Boris JP, Book DL (1973) Flux-corrected transport, I. SHASTA: A fluid transport algorithm that works. J Comput Phys 11:3869

Bryan F (1987) Parameter sensitivity of primitive equation ocean general circulation models. J Phys Oceanogr 17:970-985

Bryan K (1962) Measurements of meridional heat transport by ocean currents. J Geophys Res 67:3403-3414

Bryan K, Manabe S, Pacanowski RC (1975) A global ocean-atmosphere climate model. Part II. The oceanic circulation. J Phys Oceanogr 5:30-46

Bryan K, Lewis LJ (1979) A water mass model of the world ocean. J Geophys Res 84:2503-2517

Bryan K, Spelman MJ (1985) The ocean's response to a $\mathrm{CO}_{2^{-}}$ induced warming. J Geophys Res 90, C6:11679-11688

Colin de Verdiere A (1988) Buoyancy driven planetary flows. J Mar Res 46 (2):215-265

Cox MD (1984) A primitive equation, 3-dimensional model of the ocean. GFDL Ocean Group Tech Rep No. 1, GFDL/Princeton University USA

Cox MD (1985) An eddy resolving numerical model of the ventilated thermocline. J Phys Oceanogr 15:1312-1324

Cox MD (1987) An isopycnal diffusion in a $z$-coordinate ocean model. Ocean modelling 74:1-5 (Unpublished manuscript)

Fiadeiro ME, Veronis G (1977) On weighted-mean schemes for the finite-difference approximation to the advection-diffusion equation. Tellus 29:512-522

Gargett AE (1984) Vertical eddy diffusivity in the ocean interior. J Mar Res 42:359-393

Gent PR, McWilliams JC (1990) Isopycnal mixing in ocean circulation models. J Phys Oceanogr 20:150-155

Gerdes R (1988) Die Rolle der Dichtediffusion in numerischen Modellen der Nordatlantischen Zirkulation (in German). Ber Inst Meeresk Kiel, FRG 179:pp 176

Gordon AL (1986) Interocean exchange of thermocline water. J Geophys Res 91:5037-5046

Gregg MC (1987) Diapycnal mixing in the thermocline: a review. J Geophys Res 92:5249-5286

Hasselmann K (1982) An ocean circulation model for climate variability studies. Prog Oceanogr 11:69-92

Hellermann S, Rosenstein M (1983) Normal monthly wind stress over the world ocean with error estimates. J Phys Oceanogr 13:1093-1104

Holloway G (1989) Subgridscale representation. In: Anderson DLT, Willebrand J (eds) Modelling the ocean general circulation and geochemical tracer transport. Reidel, Dordrecht

Isemer H-J, Hasse L (1987) The Bunker climate atlas of the North
Atlantic Ocean. Vol 2, Air sea interaction. Springer, Berlin Heidelberg New York

Levitus S (1982) Climatological atlas of the world Ocean. NOAA Technical Paper 3

Luyten JR, Pedlosky J, Stommel H (1983) The ventilated thermocline. J Phys Oceanogr 13:292-309

Marotzke J, Willebrand J (1991) Multiple equilibria of the global thermohaline circulation. J Phys Oceanogr (in press)

Maier-Reimer E, Mikolajewicz U (1989) Experiments with an OGCM on the cause of the Younger Dryas. In: Ayala-Castanares, Wooster W, Yanez-Arancibia A (eds) Oceanography 1988. UNAM Press, Mexico DF, pp 87-100

Mesinger F, Arakawa A (1976) Numerical methods used in atmospheric models. GARP Publications Series No. 17, vol 1

Musgrave DL (1985) A numerical study of the roles of subgyrescale mixing and the western boundary current on homogenization of a passive tracer. J Geophys Res 90:7037-7043

O'Brien JJ (ed) (1986) Advanced physical oceanographic numerical modeling. Reidel, Hingham, Mass, USA

Olbers DJ, Wenzel M, Willebrand J (1985) The inference of North Atlantic circulation patterns from climatological hydrographic data. Rev Geophys 23:313-356

Pacanowski RC, Philander SGH (1981) Parameterization of vertical mixing in numerical models. J Phys Oceanogr 11:14431451

Redi MH (1982) Oceanic isopycnal mixing by coordinate rotation. J Phys Oceanogr 12:1154-1158

Rhines PB, Young WR (1982) Homogenization of potential vorticity in planetary gyres. J Fluid Mech 122:347-367

Roemmich D, Wunsch C (1985) Two transatlantic sections: Meridional circulation and heat flux in the subtropical North Atlantic. Deep-Sea Res 32:619-644

Rood RB (1987) Numerical advection algorithms and their role in atmospheric transport and chemistry models. Rev Geophys 25:71-100

Sarmiento JL (1986) On the north and tropical Atlantic heat balance. J Geophys Res 91:11677-11689

Sarmiento JL, Bryan K (1982) An ocean transport model for the North North Atlantic. J Geophys Res 87:394-408

Semtner AJ, Chervin RM (1988) A simulation of the global ocean circulation with resolved eddies. J Geophys Res 93: No. C12, 15502-15522

Stommel H (1948) The westward intensification of wind-driven ocean currents, Trans. Am Geophys Union 29:202-206

Weaver AJ, Sarachik ES (1990) On the importance of vertical resolution in certain ocean climate models. J Phys Oceanogr 20:600-609

Zalesak ST (1979) Fully multidimensional flux-corrected transport algorithms for fluid. J Comput Phys 31:335-362 